\title{
Falling-Incident Detection and Alarm by Smartphone with Multimedia Messaging Service (MMS)
}

\author{
Yi He, Ye Li ${ }^{*}$, Chuan Yin \\ Shenzhen Institutes of Advanced Technology, Chinese Academy of Sciences, Shenzhen, China \\ Email: *ye.li@siat.ac.cn
}

Received March 5, 2012; revised March 24, 2012; accepted March 30, 2012

\begin{abstract}
This paper presents a system with real-time classification of human movements based on smartphone mounted on the waist. The built-in tri-accelerometer was utilized to collect the information of body motion. At the same time, the smartphone is able to classify the data for activity recognition. By our algorithm, body motion can be classified into five different patterns: vertical activity, lying, sitting or static standing, horizontal activity and fall. It alarms falling by Multimedia Messaging Service (MMS) with map of suspected fall location, GPS coordinate and time etc. If a fall was suspected, an automatic MMS would be sent to pre-set people. The major advantage of the proposed system is the novel application of smartphone which already have the necessary sensors and can monitor fall ubiquitously without any additional devices.
\end{abstract}

Keywords: Multimedia Messaging Service (MMS); Smartphone; Tri-Accelerometer; Falling-Incident Detection

\section{Introduction}

Falls are the second leading cause of accidental or unintentional injury deaths world-widely. According to Centers for Disease Control and Prevention (CDC) of American, unintentional falls are a common occurrence among older adults, affecting approximately $30 \%$ of persons aged $>65$ years each year [1]. Older people have the highest risk of death or serious injury arising from a fall and the risk increases with age [2]. A "long lie” may result in negative psychological (fear of falling, which can lead to further limitation of functional activities) and physical (muscle damage, dehydration, pressure sores, hypothermia, pneumonia, and mortality) outcomes [3].

Falling-incident detection systems can be classified into computer vision [4-7] and worn devices [8-12] according to methods. For the method of computer vision, the one can be tracked by using cameras in home or other places. When the subject becomes inactive for a long time, he or she may fall. However, there are restrictions on not enough cameras in outdoor environments. On the other hand, one of the main drawbacks of current worn device solutions is that the subject has to wear an accelerometer and many elder people forget to put on or refuse such devices [4].

Falling-incident detection using a mobilephone is a feasible and highly attractive technology for elder adults, especially those living alone [13]. According to Interna-

*Corresponding author. tional Telecommunication Union (ITU), the number of mobile phone subscriptions worldwide has reached 4.6 billion by the end of 2009. Mobile phone is one fast growing device which becomes indispensable to people almost every moment, whereas users may forget to wear special micro sensors. Using off-the-shelf mobile phones, not only reduces the cost, also exploits skilled hardware and ubiquitous monitor.

Previous studies have also demonstrated the effectiveness of using mobile phone to detect fall. For example, T. Zhang et al. [14] proposed an application to detect falls by embedding an accelerometer in cell phone and using KFD algorithm. J. Yang [15] performed physical motion recognition using mobile phones, i.e. Nokia N95, with built-in accelerometer sensors. T. Saponas et al. [16] classified human activities in real time using three-axis accelerometer of the Apple iPhone and the Nike + iPod Sport Kit. R. Y. W. Lee et al. [13] used two devices, i.e. a mobile phone and an independent accelerometer, attached to the waist of the participants to study the sensitivity and specificity of falling-incident detection. All of above sent Short Messaging Service (SMS) with fall time and fall location. However, this paper warns fall using Multimedia Messaging Service (MMS) included with time, map of suspected fall location and GPS coordinate etc. And this paper presents an implementation of a real-time classification of human movement based on smart phone mounted on the waist.

The remainder of this paper is as follows. In Section 2, 
the methods of data collection and data preprocessing are introduced, followed by the feature extraction and classification algorithm. And then the fall location and alarm with MMS are described. The experimental results are given in Section 3. We conclude the work in Section 4 with suggestions to future work.

\section{Methods}

Lenovo Le-Phone is a smartphone which carrys Android operating system. Le-Phone packs a $1 \mathrm{GHz}$ Snapdragon CPU, a 3.7" AMOLED capacitive touch screen with WVGA resolution. As illustrated in Figure 1, Le-Phone with built-in tri-accelerometer was utilized to collect the signal which represents the body movement. At the same time, the smart phone classifies the data to recognize fall. If a fall was suspected, an automatic MMS would be sent to guardian with information including the time, GPS coordinate, the map of suspected fall location.

\subsection{Data Collection}

Head and waist are relevant sites for accelerometer detection of falls, using simple thresholds and posture detection [17]. So phone in this system is worn on the waist, as show in Figure 2.

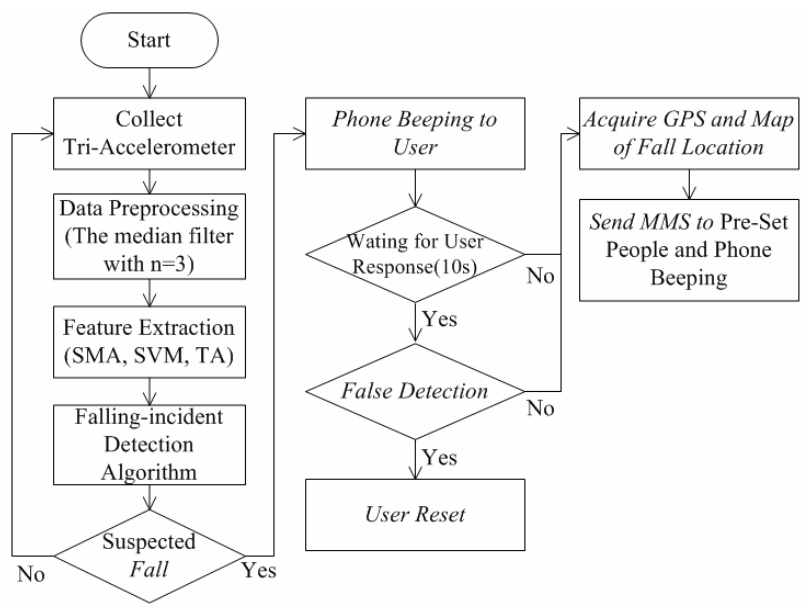

Figure 1. A block diagram of the falling-incident detection system based on Le-Phone.

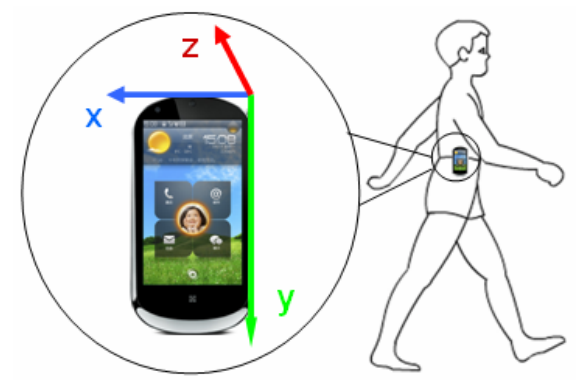

Figure 2. The position of wear mobile phone and the coordinate of fall detect system.
Because essentially all measured body movements are contained with in frequency components below $20 \mathrm{~Hz}$ (indeed, even in gait, 99\% of the energy is contained below $15 \mathrm{~Hz}$ ) [8]. Lenovo Le-Phone with Android operating system is used to acquire tri-accelerometer signals sampled at $40 \mathrm{~Hz}$. As displayed in Figure 3, X-axis, $\mathrm{Y}$-axis and Z-axis data of accelerometer were collected by the Le-Phone.

\subsection{Data Preprocessing}

The output of an ideal accelerometer worn on the human body originates from several sources: 1) acceleration due to body motion; 2) gravitational acceleration; 3) external vibrations, not produced by the body itself (e.g., resulting from vehicles); 4) accelerations due to bouncing of the sensor against other objects, eventually resulting in mechanical resonance [18]. Sources 1 and 2 are directly related to intentional movement of the body. However, sources 3 and 4, which may add "noise" to the accelerometer output, should be attenuated by adequate filtering techniques. The median filter with $n=3$ is applied to the raw data to attenuate the noise. As Indicated in Figure 4, median filter has played a nice smoothing effect. $m_{\text {Zraw }}$, $\sigma_{\text {Zraw }}$ are mean and standard deviation of raw Z-axis data from the static phone on the desk respectively.

$$
\left(m_{Z_{\text {raw }}}, \sigma_{\text {Zraw }}\right)=(9.7409,0.1008)
$$

while $m_{Z \text { smooth }}, \sigma_{Z s m o o t h}$ are mean and standard deviation of raw Z-axis data after median filter respectively.

$$
\left(m_{Z \text { smooth }}, \sigma_{Z \text { smooth }}\right)=(9.7441,0.0502)
$$

\subsection{Feature Extraction}

1) Signal Magnitude Area (SMA) [18]

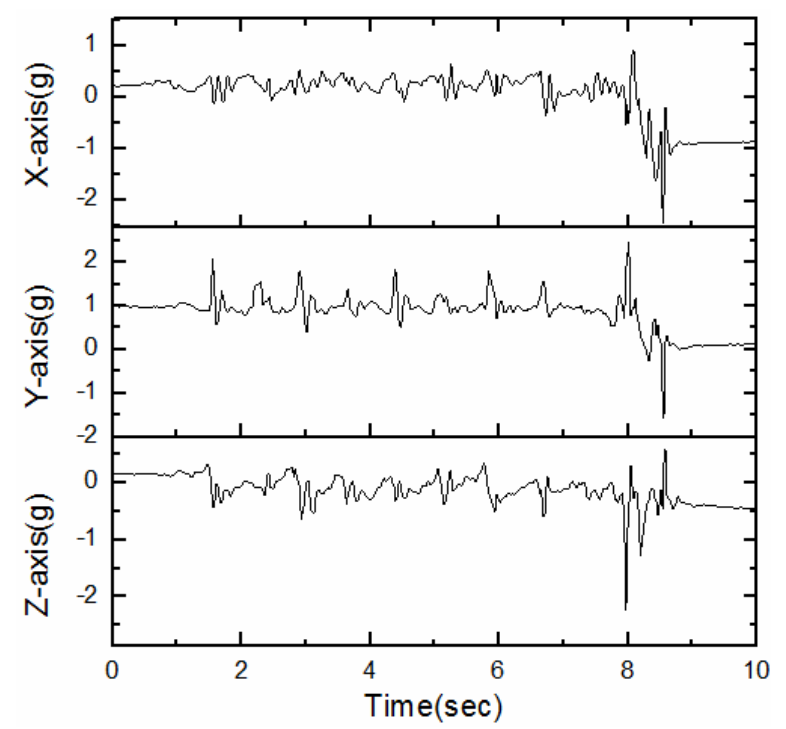

Figure 3. Data collection by Le-Phone. 


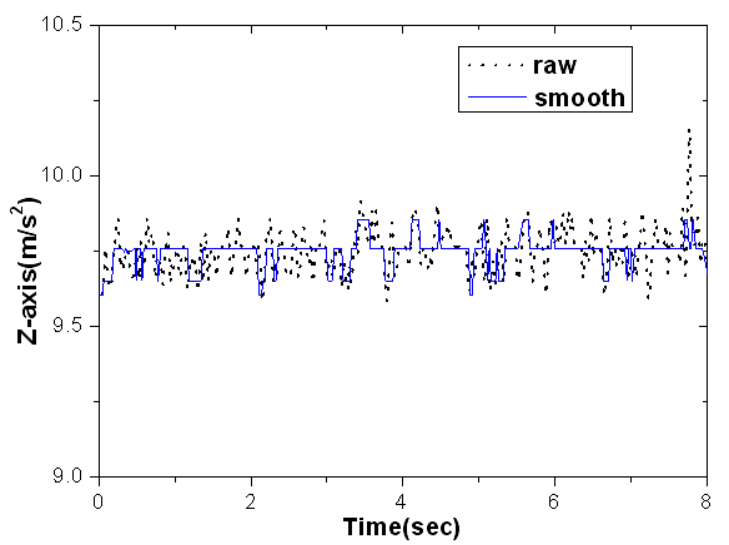

Figure 4. Raw Z-axis data and smooth Z-axis data after median filter from the static phone on the desk.

SMA [defined in (3)] is utilized to distinguish between periods of user activity and rest [8]. SMA characterizes the degree of change of human movement, the greater the value that the more violent motion state changes.

$$
\mathrm{SMA}=\frac{1}{t}\left(\int_{0}^{t}|x(t)| \mathrm{d} t+\int_{0}^{t}|y(t)| \mathrm{d} t+\int_{0}^{t}|y(t)| \mathrm{d} t\right)
$$

where $x(t), y(t)$, and $z(t)$ refer to the tri-accelerometer signals of the $\mathrm{x}-, \mathrm{y}-$, and $\mathrm{z}$-axis samples, respectively.

2) Signal Magnitude Vector (SVM)

$$
\mathrm{SVM}=\sqrt{x_{i}^{2}+y_{i}^{2}+z_{i}^{2}}
$$

where $x_{i}$ is the $i$ th sample of the $\mathrm{x}$-axis signal (similarly for $y_{i}$ and $z_{i}$ ). The test results identified the most suitable parameter to be SVM at a threshold of $2.5 \mathrm{~g}$ (as shown in Figure 7). When an SVM value was above the threshold, fall could be deemed to have occurred.

3) Tilt Angle (TA)

Postural orientation refers to the relative tilt of the body in space. As indicated in (5), the tilt angle of user was defined as the angle between the positive y-axis and the gravitational vector $g$ by the relation:

$$
\mathrm{TA}=\arcsin \left(\frac{y_{i}}{\sqrt{x_{i}^{2}+y_{i}^{2}+z_{i}^{2}}}\right)
$$

If TA was below the threshold 40 degree (as shown in Figure 7), it was classified as fall or lie.

\subsection{Classification Algorithm}

This flowchart (as shown in Figure 5) displays the major steps of the algorithm embedded into Le-Phone. Body motion was successfully recognized as five different patterns: vertical active, lying, sitting or static standing, horizontal active and fall.

\subsection{Location and Alarm}

As illustrated in Figure 6, Map of suspected fall location

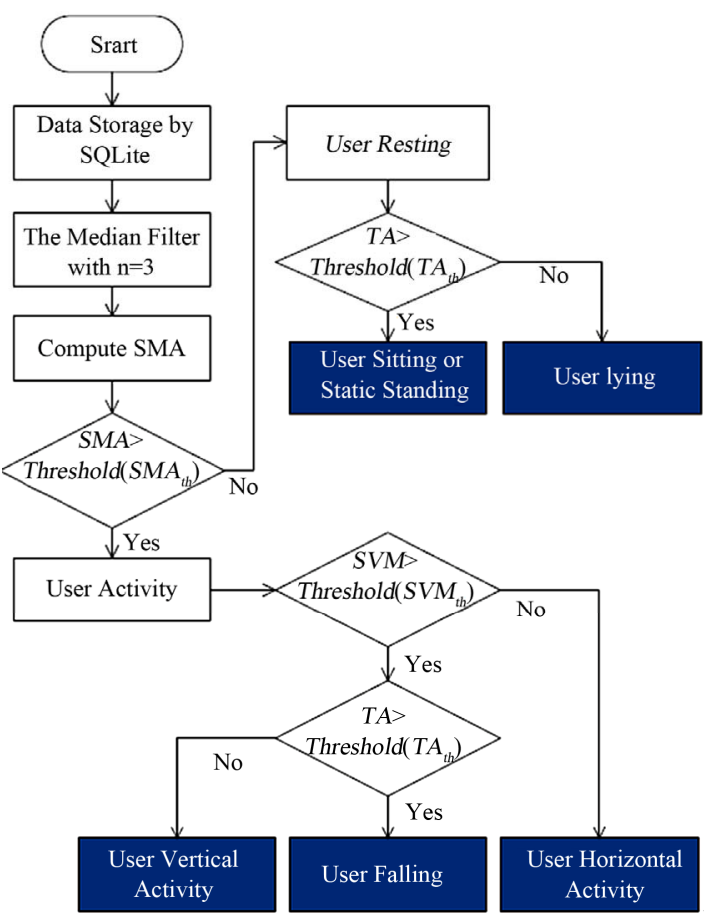

Figure 5. Falling-incident detection algorithm.

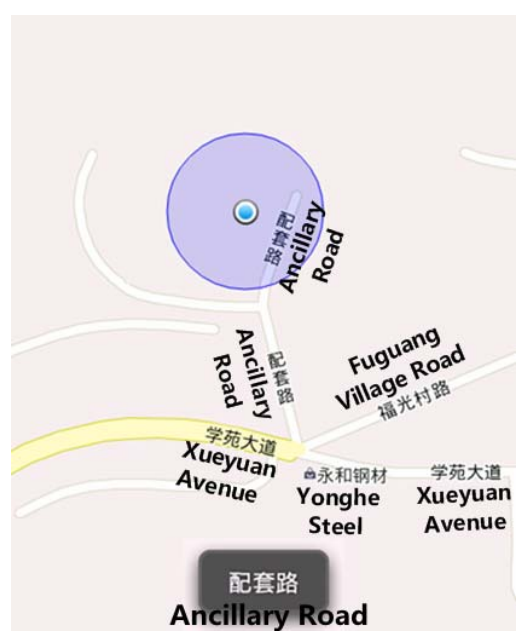

Figure 6. Map of suspected fall location.

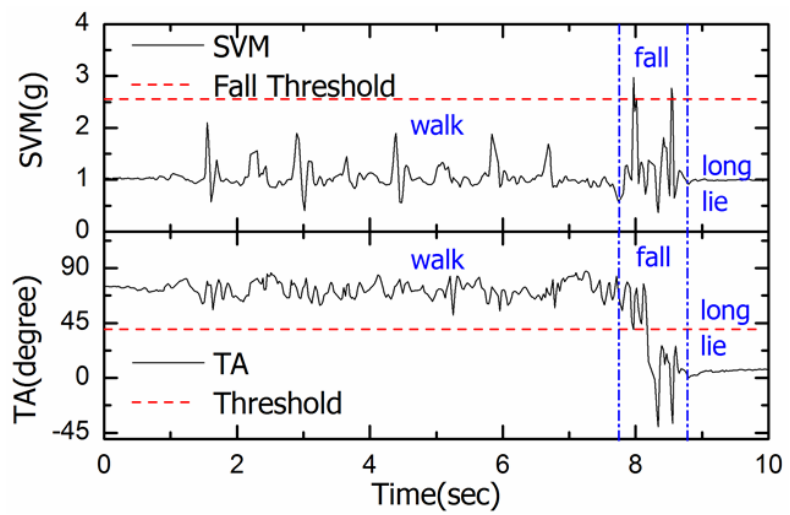

Figure 7. Comparison of the magnitude of SVM and TA. 
was acquired and a part of MMS would be sent to pre-set guardian. In the map, the blue point represents the suspected fall location. And at the bottom of the map, the fall address was displayed.

\section{Results}

As indicated in Figure 7, when SMA, SVM and TA are above the respective threshold, a fall was suspected. In addition, MMS included with map of suspected fall location, GPS coordinate and time was sent to pre-selected guardian.

\section{Conclusions}

The falling-incident detection system based on the data acquired from a waist-mounted smartphone was developed for use in a real-time environment. By our algorithm, body motion can be classified into five different patterns: vertical activity, lying, sitting or static standing, horizontal activity and fall. The major advantage of the proposed solution is that smartphone is readily available to most people and users may forget to wear other special micro sensors but not smartphone. In addition, the MMS of positioning the fall location automatically encourages the elderly to exercise outdoors.

However, there are two problems we need to further study to make the solution more feasible. One is the problem of adaptation to daily wear. The system requests the smart phone to be worn on the waist. However, normally people wear phones in the pocket of shirts or pants, which would cause loose attachment between the body and smartphone, resulting in mechanical movement eventually. Another problem is to detect falls anytime. The system would be working only when the smartphone is mounted on the waist. Assume that the user falls when he is making a phone call or message reading, the system would not be able to detect falls in such scenarios.

\section{Acknowledgements}

This work was supported in part by Shenzhen Basic Research Funds for Distinguished Young Scientists (No.JC 201005270306A), Guangdong Province \& Chinese Academy of Sciences Comprehensive Strategic Cooperation Project (No.2011A090100025) and Shenzhen Basic Research Project (No. JC201005270258A).

\section{REFERENCES}

[1] J. M. Hausdorff, D. A. Rios and H. K. Edelberg, "Gait Variability and Fall Risk in Community-Living Older Adults: A 1-Year Prospective Study," Archives of Physical Medicine and Rehabilitation, Vol. 82, No. 8, 2001, pp. 1050-1056. doi:10.1053/apmr.2001.24893

[2] W. H. O. Ageing and L. C. Unit, "WHO Global Report on Falls Prevention in Older Age,” World Health Organization, Geneva, 2008.

[3] S. R. Lord, C. Sherrington and H. B. Menz, "Falls in Older People: Risk Factors and Strategies for Prevention,” Cambridge University Press, Cambridge, 2007. doi:10.1017/CBO9780511722233

[4] B. Schulze, M. Floeck and L. Litz, "Concept and Design of a Video Monitoring System for Activity Recognition and Fall Detection,” Computer Science, Vol. 5597, 2009, pp. 182-189.

[5] A. Sixsmith and N. Johnson, "A Smart Sensor to Detect the Falls of the Elderly," IEEE Pervasive Computing, Vol. 3, No. 2, 2004, pp. 42-47. doi:10.1109/MPRV.2004.1316817

[6] M. Yu, S. M. Naqvi, A. Rhuma and J. Chambers, "Fall Detection in a Smart Room by Using a Fuzzy One Class Support Vector Machine and Imperfect Training Data," IEEE International Conference on Acoustics, Speech and Signal Processing, 22-27 May 2011, Prague, pp. 18331836.

[7] J. Ben-Arie, Z. Wang, P. Pandit and S. Rajaram, "Human Activity Recognition Using Multidimensional Indexing," IEEE Transactions on Pattern Analysis and Machine Intelligence, Vol. 24, No. 8, 2002, pp. 1091-1104. doi:10.1109/TPAMI.2002.1023805

[8] D. M. Karantonis, M. R. Narayanan, M. Mathie, N. H. Lovell and B. G. Celler, "Implementation of a Real-Time Human Movement Classifier Using a Triaxial Accelerometer for Ambulatory Monitoring," Information Technology in Biomedicine, Vol. 10, No. 1, 2006, pp. 156-167. doi:10.1109/TITB.2005.856864

[9] T. Choudhury, G. Borriello, S. Consolvo, D. Haehnel, B. Harrison, B. Hemingway and J. Hightower, “The Mobile Sensing Platform: An Embedded Activity Recognition System,” IEEE Pervasive Computing, Vol. 7, No. 2, 2008, pp. 32-41. doi:10.1109/MPRV.2008.39

[10] H. Ghasemzadeh, R. Jafari and B. Prabhakaran, “A Body Sensor Network with Electromyogram and Inertial Sensors: Multimodal Interpretation of Muscular Activities," Information Technology in Biomedicine, Vol. 14, No. 2, 2010, pp. 198-206. doi:10.1109/TITB.2009.2035050

[11] I. Maglogiannis and C. Doukas, "Emergency Fall Incidents Detection in Assisted Living Environments Utilizing Motion, Sound and Visual Perceptual Components," Information Technology in Biomedicine, Vol. 15, No. 2, 2011, pp. 277-289.

[12] E. M. Tapia, S. S. Intille, W. Haskell, K. Larson, J. Wright, A. King and R. Friedman, "Real-Time Recognition of Physical Activities and Their Intensities Using Wireless Accelerometers and a Heart Rate Monitor,” 11th IEEE International Symposium on Wearable Computers, Boston, 11-13 October 2007, pp. 37-40.

[13] R. Y. W. Lee and A. J. Carlisle, "Detection of Falls Using Accelerometers and Mobile Phone Technology," Age and Ageing, Vol. 40, 2011, pp. 690-696.

[14] T. Zhang, J. Wang, P. Liu and J. Hou, "Fall Detection by Embedding an Accelerometer in Cellphone and Using KFD Algorithm," International Journal of Computer Science and Network Security, Vol. 6, No. 3, 2006, pp. 
277-284.

[15] J. Yang, “Toward Physical Activity Diary: Motion Recognition Using Simple Acceleration Features with Mobile Phones," Proceedings of the 1st International Workshop on Interactive Multimedia for Consumer Electronics, New York, 23 October 2009, pp. 1-10.

[16] T. Saponas, J. Lester, J. Froehlich, J. Fogarty and J. Landay, "iLearn on the iPhone: Real-Time Human Activity Classification on Commodity Mobile Phones," University of Washington, Washington, 2008.
[17] M. Kangas, A. Konttila, I. Winblad and T. Jamsa, "Determination of Simple Thresholds for AccelerometryBased Parameters for Fall Detection,” Proceedings of the 29th Annual International Conference of the IEEE EMBS, Lyon, 22-26 August 2007, pp. 1367-1370.

[18] C. V. C. Bouten, K. T. M. Koekkoek, M. Verduin, R. Kodde and J. D. Janssen, “A Triaxial Accelerometer and Portable Data Processing Unit for the Assessment of Daily Physical Activity,” Biomedical Engineering, Vol. 44, No. 3, 1997, pp. 136-147. doi:10.1109/10.554760 